Abstract: We present an overview of our results concerning the influence of two-pair continuum coherences on the transient nonlinear optical response of semiconductor microcavities. We show that the interplay between these 4-particle coherences and the nonperturbative light-matter interaction produces highly desirable almost decoherence-free exciton-exciton collisions on the lower polariton branch. This effect gives rise to the very different nonlinear absorption rates on the two polariton branches observed in many experiments and make possible to reach a very high degree of amplification in samples with large Rabi splitting. Moreover, we show that the availability of almost decoherence free exciton-exciton collisions can be used for the realization of coherent trapping of polariton emission and amplification. This coherent manipulation and trapping of many-particle polariton states can be performed employing both ultrafast and continuous wave operation.

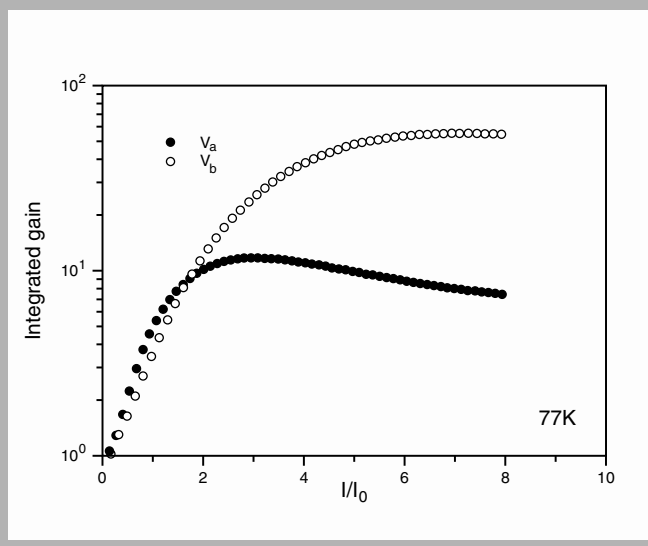

The power dependence of the integrated gain

\title{
Coherence and correlation in semiconductor microcavities
}

\author{
O. Di Stefano, S. Savasta, and R. Girlanda*
}

Dipartimento di Fisica della Materia e Tecnologie Fisiche Avanzate, Universita di Messina, Salita Sperone 31,1-98166 Messina, Italy

Key words: excitons; polaritons; nonlinear optics; semiconductor microcavities

\section{Introduction}

A semiconductor microcavity (SMC) is a photonic structure designed to enhance light-matter interactions. The strong light-matter interaction in these systems gives rise to cavity polaritons which are hybrid quasiparticles consisting of a superposition of cavity photons and collective electronic excitations (excitons). The dynamics and hence the resulting energy bands of these mixed quasiparticles are highly distorted respect to those of bare excitons and cavity photons [1].

It has been realized by a number of groups that polariton parametric scattering in microcavities provides for a very efficient source of light amplification. Already in the pioneering work of Ref. [2] an impressive gain of up to about 100 for a single pass through a quantum well microcavity was reported and has been interpreted as a coherent parametric scattering of polaritons. In recent experiments even a gain of 5000 has been demonstrated [3].

Exciton systems are a good candidate as coherent amplifiers owing to the very large optical nonlinearities that they support when they are resonantly driven. Unfortunately exciton-exciton $(X X)$ collisions are affected by decoherence producing excitation induced dephasing (EID), hence increasing the system pumping also increases decay rates (EID), efficient amplification would be prevented.

Here we show that the unexpected increase observed in experiments is a direct consequence of many-body correlations beyond mean-field. We show that the possibility of observing very large gains is a consequence of the coherent control that the exciton-photon interaction exerts on the non-instantaneous exciton-exciton collisions [4]. From this analysis it clearly follows that the memory structures induced by $X X$ continuum correlations can

* Corresponding author: e-mail: Raffaello.Girlanda@unime.it 
be used actively to manipulate the response. In particular, by engineering the polariton dispersion in a microcavity it is possible to tune the amplification properties of the system by orders of magnitude. Moreover this control is able to produce almost decoherence-free exciton-exciton collisions of great importance for the development of future all-optical devices [5]. For example we show that the availability of almost decoherence free exciton-exciton collisions can be used for the realization of coherent trapping of polariton emission and amplification.

The coherent trapping effect here presented has a working principle very close to coherent population trapping in three-level $\Lambda$-type atoms (See e.g. [6]). We can trap the populations of pump polaritons so that no population transfer to signal and idler may occur despite the presence of real stimulation felt by pump polaritons. This coherent manipulation and trapping of many-particle polariton states can be performed employing both ultrafast and continuous wave operation. Both the presence of strong correlation effects as well as the formation of coherences in these nonlinear optical devices may have important implications for the controllability of optical signals, which are of great importance for applications in present-day and future optoelectronic devices.

\section{Many-body and correlation effects in semiconductor microcavities}

The system that we investigate consists of one or more QWs grown inside a wavelength scale semiconductor planar Fabry-Perot. A weak probe signal pulse injected perpendicular to the cavity (in-plane wave vector $\mathbf{k}=0$ ) can be greatly amplified by means of a parametric process if a pump beam seeds the SMC at a specific angle (the magic angle corresponding to the inflection point of the polariton dispersion). In particular the weak signal at normal incidence stimulates the scattering of pump polariton pairs into different pairs. The emitted pairs are constituted by a signal polariton $(\mathbf{k}=0)$ and a higher energy polariton usually named idler. The magic angle is the one ensuring energy and momentum conservation for pair scattering. The in-plane momentum conservation requires that the idler momentum be twice the momentum of pump polaritons (signal polaritons have zero in-plane momentum) and energy conservation can be expressed as $2 \omega_{k}=\omega_{0}+\omega_{2 k}$, where $\omega_{k}$ is the energy of a pump polariton injected with an in-plane wavevector $\mathbf{k}$, and $\omega_{2 k}$ is the energy of the idler polariton. We observe that energy conservation for this process is allowed by the peculiar shape of the lower polariton energy dispersion as a function of the in-plane momentum $k$. Thanks to the strong polaritonpolariton scattering rates and to the feedback provided by the cavity, the emitted pairs contribute to the stimulation of new pair scattering events from the pump, giving rise to giant amplification in close analogy with the laser action. This final state stimulation is evidenced by the exponential power dependence of the gain on the pump power.
We focus on the case of input light beams with all the same circularly (e.g. $\sigma_{+}$) polarization, thus excluding the coherent excitation of bound two-pair coherences (biexcitons). We start from the Heisenberg equations for the exciton polarization and cavity field describing quantum optical effects and coherent nonlinear optics in SMCs [7], where the hierarchy of higher order density matrices has been closed by invoking the dynamics controlled truncation (DCT) scheme [8]. The variables describing two-pair transitions can be eliminated by formally inverting the corresponding equations of motion. This results in a memory kernel representation of the two-pair transitions that is nonlocal in time and enters the equation of motion of single pair transition densities. The intracavity light-field is described within the quasi-mode scheme [7]. The time evolution of the coupled $1 \mathrm{~s}$ exciton $\left(\mathrm{P}_{k}\right)$ and photon waves $\left(E_{k}\right)$ including finite duration of $X X$ collisions [4,5] obey the following set of coupled equations,

$$
\begin{aligned}
\frac{\partial}{\partial t} E_{\mathbf{k}} & =-\left(\gamma_{c}+i \omega_{k}^{c}\right) E_{\mathbf{k}}+i V P_{\mathbf{k}}+t_{c} E_{\mathbf{k}}^{i n}, \\
\frac{\partial}{\partial t} B_{\mathbf{k}} & =-\left(\gamma_{x}+i \omega_{x}\right) P_{\mathbf{k}}+i V E_{\mathbf{k}}-i R_{\mathbf{k}}^{N L},
\end{aligned}
$$

where $\omega_{k}^{c}, \omega_{x}$, and $\gamma_{c}, \gamma_{x}$ are the energies and dephasing rates of cavity photons and QW excitons respectively. $E_{\mathbf{k}}^{i n}$ describes input light pulses (more precisely $\left|E_{\mathbf{k}}^{i n}\right|^{2}$ gives the input photon rate per unit area), $t_{c}$ determines the beam fraction passing the cavity mirror $\left(t_{c}=\sqrt{\gamma_{c}}\right.$ for a cavity with equal mirrors). The intracavity photon-field and the exciton field of a given mode $\mathbf{k}$ are coupled by $V$. The polariton splitting in SMCs can be increased by inserting a large number of QWs into the cavity $\left(V=V_{1} \sqrt{N_{\text {eff }}}\right.$, where $V_{1}$ is the exciton-photon coupling for $1 \mathrm{QW}$ and the effective number of QWs $N_{\text {eff }}$ depends on the number of wells inside the cavity and their spatial overlap with the cavity-mode). The relevant nonlinear source term, able to couple waves with different in-plane wave-vector $\mathbf{k}$, is given by $R_{\mathbf{k}}^{N L}=\left(R_{\mathbf{k}}^{s a t}+R^{X X}\right) / N_{e f f}$, where the first term originates from the phase-space filling of the exciton transition,

$R_{\mathbf{k}}^{s a t}=\frac{V}{n_{s a t}} \sum_{\mathbf{k}^{\prime} \mathbf{k}^{\prime \prime}} P_{q}^{*} P_{\mathbf{k}^{\prime \prime}} E_{\mathbf{k}^{\prime}}$,

being $n_{\text {sat }}=7 /\left(4 \pi a_{0}^{2}\right)$ the exciton saturation density $\left(\mathbf{k}^{\prime}, \mathbf{k}^{\prime \prime}\right.$, and $\mathbf{q}$ are tied by the momentum conservation relation $\left.\mathbf{k}+\mathbf{q}=\mathbf{k}^{\prime}+\mathbf{k}^{\prime \prime}\right), a_{0}$ is the exciton Bohr radius determining the extension of the $1 \mathrm{~s}$ wavefunction: $\Phi_{x}(r)=2 \sqrt{2 / \pi a_{0}^{2}} \exp \left(-2 r / a_{0}\right) . R_{\mathbf{k}}^{X X}$ is the Coulomb interaction term. It dominates the coherent $X X$ coupling and for co-circularly polarized waves can be written as

$$
\begin{aligned}
& R_{\mathbf{k}}^{X X}=\sum_{\mathbf{k}^{\prime} \mathbf{k}^{\prime \prime}} V_{X X} P_{\mathbf{q}}^{*}(t) P_{\mathbf{k}^{\prime \prime}}(t) P_{\mathbf{k}^{\prime}}(t)- \\
& -i P_{\mathbf{q}}^{*}(t) \int_{-\infty}^{t} F\left(t-t^{\prime}\right) P_{\mathbf{k}^{\prime \prime}}\left(t^{\prime}\right) P_{\mathbf{k}^{\prime}}\left(t^{\prime}\right) d t^{\prime} .
\end{aligned}
$$


$R_{\mathrm{k}}^{X X}$ includes the instantaneous mean-field $X X$ interaction term $V_{X X} \simeq 1.52 E_{b} a_{0}^{2}$ ( $E_{b}$ is the exciton binding energy) plus a non instantaneous term originating from 4particle correlations. This coherent memory can be interpreted as a non-markovian process involving the 2-particle (excitons) polarization waves interacting with a bath of 4particle correlations $[9,10]$. The memory kernel $F(\tau)$ can be written in the following form,

$F(\tau)=\Theta(\tau) \sum_{m}\left|\left\langle 0|\hat{D}| E_{m}\right\rangle\right|^{2} e^{-\left(i \omega_{m}+\Gamma\right) \tau}$,

where $\Theta(\tau)$ is the step function, $\hat{D}=\left[\hat{B},\left[\hat{B}, \hat{V}_{c}\right]\right](\hat{B}$ is the exciton destruction operator, while $\hat{V}_{c}$ is the Coulomb interaction operator), and $\left|E_{m}\right\rangle$ and $\omega_{m}$ describe the eigenstates and the corresponding continuous spectrum of energies of the 4-particle Hilbert subspace [5,10,11]. $\Gamma$ describes the dephasing of two pair coherences (in the following we will use $\left.\Gamma=2 \pi \gamma_{x}\right)$. We calculated $F(\tau)$ for QW excitons following a recent microscopic approach $[12,13]$ based on the T-matrix. By this approach the 4particle states $\left|E_{m}\right\rangle$ are expanded in terms of exciton-pairs on the 1s parabola. We calculated $F(\tau)$ for a $7 \mathrm{~nm} \mathrm{GaAs}$ QW with binding energy $E_{b}=13.5 \mathrm{meV}$. Defining

$\mathcal{F}(\omega)=V_{X X}+\int_{-\infty}^{\infty} d t F(t) e^{i \omega t}$.

We observe that the imaginary part of $\mathcal{F}(\omega)$ reflects the density of states of 2-exciton pair coherences.

In order to obtain from the coupled exciton photon system the dynamics for the polariton system we express Eq. (1) in compact form

$i \dot{\mathcal{B}}_{\mathbf{k}}=\mathcal{H}_{\mathbf{k}} \mathcal{B}_{\mathbf{k}}+\tilde{\mathcal{R}}_{\mathbf{k}}^{N L}$

where

$\mathcal{B}_{\mathbf{k}}=\left(\begin{array}{c}B_{\mathbf{k}} \\ E_{\mathbf{k}}\end{array}\right), \mathcal{H}_{\mathbf{k}}=\left(\begin{array}{cc}\omega_{x} & -V \\ -V & \omega_{\mathbf{k}}^{c}\end{array}\right), \quad$ and

$\tilde{\mathcal{R}}_{\mathbf{k}}^{N L}=\left(\begin{array}{c}\mathcal{R}_{\mathbf{k}}^{N L} \\ 0\end{array}\right)$.

Now we perform the unitary basis transformation:

$P_{j \mathbf{k}}=X_{j \mathbf{k}} B_{\mathbf{k}}+C_{j \mathbf{k}} E_{\mathbf{k}}$.

After few algebra we obtain the polariton dynamic equations

$\dot{P}_{j \mathbf{k}}==-i \Omega_{1 \mathbf{k}} \hat{P}_{j \mathbf{k}}+E_{j \mathbf{k}}-i \Omega_{j \mathbf{k}}^{N L}$,

where $E_{j \mathbf{k}}=t_{c} C_{j \mathbf{k} E_{\mathbf{k}}}^{i n}$ and $\Omega_{j \mathbf{k}}^{N L}=X_{j \mathbf{k}} \mathcal{R}_{\mathbf{k}}^{N L}$. If we limit to the lower polariton branch, straightforward calculation gives for the nonlinear term

$\mathcal{R}_{\mathbf{k}}^{N L}=$ $=\sum_{\mathbf{k}_{1}, \mathbf{k}_{2}, \mathbf{k}_{3}} \int_{-\infty}^{\infty} g_{\mathbf{k}_{1}, \mathbf{k}_{2}, \mathbf{k}_{3}}\left(t, t^{\prime}\right) \hat{P}_{1 \mathbf{k}}^{\dagger}(t) \hat{P}_{1 \mathbf{k}}\left(t^{\prime}\right) \hat{P}_{1 \mathbf{k}}\left(t^{\prime}\right) d t^{\prime}$,

being

$g_{\mathbf{k}_{1}, \mathbf{k}_{2}, \mathbf{k}_{3}}\left(t, t^{\prime}\right)=$

$=\left[V_{X X} \delta\left(t-t^{\prime}\right)-i F\left(t-t^{\prime}\right)\right] X_{1 \mathbf{k}_{1}}^{*} X_{1 \mathbf{k}_{2}} X_{1 \mathbf{k}_{3}}$.

Eq. (7) clearly displays the structure of the polaritonpolariton scattering mechanism: $R_{\mathbf{k}}^{N L}$ couples polaritons waves belonging to different $\mathbf{k}$ modes. We also observe that the strong exciton-photon coupling does not modify the memory kernel $F(\tau)$ as a consequence of the fact that 4-particle correlations do not couple to cavity photons $[7,5,13]$. However cavity effects are able to alter the phase dynamics of the 2-particle polarization waves $P_{\mathbf{k}}$ during collisions, i.e. on a timescale shorter than the decay time of the memory kernel $F(\tau)$. In particular the phase of 2particle polarization waves in SMCs oscillates with a frequency (fixed by the polariton dispersion relations) modified respect to that of excitons in bare QWs. This fact can produce a deep modification of the integral in Eq. (3). This mechanism can be more clearly understood simplifying the integral in Eq. (3). Inserting Eq. (4) into Eq. (3), the resulting integral term resembles the corresponding term determining the spontaneous decay of a two-level transition (See e.g. [14]). It is possible to simplify the integral in Eq. (3) following this analogy. We can write the polarization densities as $P_{\mathbf{k}}(t)=Y_{\mathbf{k}}(t) \exp \left(-i \omega_{k} t\right)$ and can assume that the amplitudes $Y_{\mathbf{k}}(t)$ are slowly varying functions of time as compared to the ultrafast decay time of the memory function $F(\tau)$. Then, adopting the wellknown Weisskopf-Wigner approximation used to analyze the spontaneous emission between two atomic levels, the dominant $X X$ interaction term for the signal (0) and idler (2k) modes can be written as [5]

$R_{0(2 \mathbf{k})}^{X X}=$

$=2 \mathcal{F}\left(\omega_{0(2 \mathbf{k})}+\omega_{\mathbf{k}}\right)\left|X_{\mathbf{k}}\right|^{2} X_{0(2 \mathbf{k})}\left|P_{\mathbf{k}}(t)\right|^{2} P_{0(2 \mathbf{k})}(t)+$

$+\mathcal{F}\left(2 \omega_{\mathbf{k}}\right) X_{2 \mathbf{k}(0)}^{*} X_{\mathbf{k}}^{2} P_{2 \mathbf{k}(0)}^{*}(t) P_{\mathbf{k}}^{2}(t)$.

The spontaneous emission rate of atoms, according to the Weisskopf-Wigner theory depends on the optical density of states calculated at the transition energy. Analogously Eq. (9) shows that the $X X$ scattering rates depend on the spectral density of two pair transitions calculated at the energy of the colliding polariton pairs. The first term (arising from self-scattering of polariton pairs) produces a blue-shift of the polariton resonance and introduces an intensity-dependent dephasing mechanism. The second term provides the coupling mechanism able to transfer polaritons from the pump to the signal and idler modes. This term describes the scattering of a polariton pair (2 pump polaritons) into a different pair (the signal plus the idler) with pair energy and momentum conservation. An analogous expression can be derived for the exciton-exciton interaction of the pump mode. This 
Weisskopf-Wigner description of 4-particle correlation effects in SMCs sets out the relevance of polariton pairs in the scattering process. The obtained 4-particle spectral density displays strong variations within the spectral region of interest around $2 \omega_{0}$. In particular, moving towards the low energy region, the dispersive part $\operatorname{Re}(\mathrm{F})$ increases while the absorptive part $\operatorname{Im}(\mathcal{F})$ that contrasts gain goes to zero. As a consequence, the exciton-exciton scattering rates in SMCs differ significantly from those of resonantly excited excitons in bare QWs. Mean-field calculations do not take into account this difference. We observe that (i) these spectral variations of $\mathcal{F}(\omega)$ origins from the finite duration of the $X X$ interaction (instantaneous $X X$ collisions would have implied the absence of spectral variations of $\mathcal{F}(\omega)$ ). (ii) Owing to the spectral behavior of $\mathcal{F}(\omega)$, changing the polariton resonances, e.g. by changing the polariton splitting or varying the detuning (the energy difference between the cavity and exciton resonances) it is possible to alter profoundly the scattering rates.

The Weisskopf-Wigner approximation just provided a useful guideline to understand the experimental findings. In the following we present numerical results obtained solving the system of 6 integro-differential equations for three modes: the signal (at normal incidence), the pump ( $k$ generate by light seeding the cavity at the magic angle of incidence such that $2 \Omega_{k}=\Omega_{0}+\Omega_{2 k}$ and the idler $(2 \mathbf{k})$. The microscopic theory of polariton parametric amplification here presented allows a fully quantitative analysis of recent measurements. The gain curves versus the input pump power calculated for GaAlAs-based samples with splitting $2 V_{a}=10.6 \mathrm{meV}\left(N_{e f f}=4\right)$ and $2 V_{b}=15 \mathrm{meV}$ $\left(N_{\text {eff }}=8\right)$ (Fig. 1), fully confirm the analysis based on the Weisskopf-Wigner theory. The calculated gain is defined as the total light intensity transmitted in the signal direction divided by the intensity transmitted in the absence of the pump beam. An increase of less then a factor $1 / 3$ in the polariton splitting produces an increase of the maximum achievable gain at $T=10 \mathrm{~K}$ (Fig. 1, upper panel) of more than one order of magnitude (Fig. 1, upper panel) in close agreement with experimental results [3]. Fig. 1 (lower panel) shows that an increase of less then a factor $1 / 3$ in the polariton splitting produces an increase of the maximum achievable gain at $T=77 \mathrm{~K}$ of six time in agreement with experimental results [3]. This result shows how the increase of the exciton-photon coupling favours high-temperature operation. The power dependence of gain shows an almost exponential growth and then saturates at high powers. The saturation of gain is mainly determined by the nonlinear absorption that is most relevant for the idler beam (according to Eq. (9) the idler nonlinear absorption is determined by $\operatorname{Im} \mathcal{F}\left(\omega_{2 \mathbf{k}}+\omega_{\mathbf{k}}\right)$. We observe that the increase of the idler nonlinear absorbance is highly superlinear because the increase of the pump power produces both a direct increase of the exciton density and an increase of $\operatorname{Im} \mathcal{F}\left(\omega_{2 \mathbf{k}}+\omega_{\mathbf{k}}\right)$ as a consequence of the blue shift of the polariton-pair resonance $\omega_{2 \mathbf{k}}+\omega_{\mathbf{k}}$ induced by $\operatorname{Re} \mathcal{F}$.
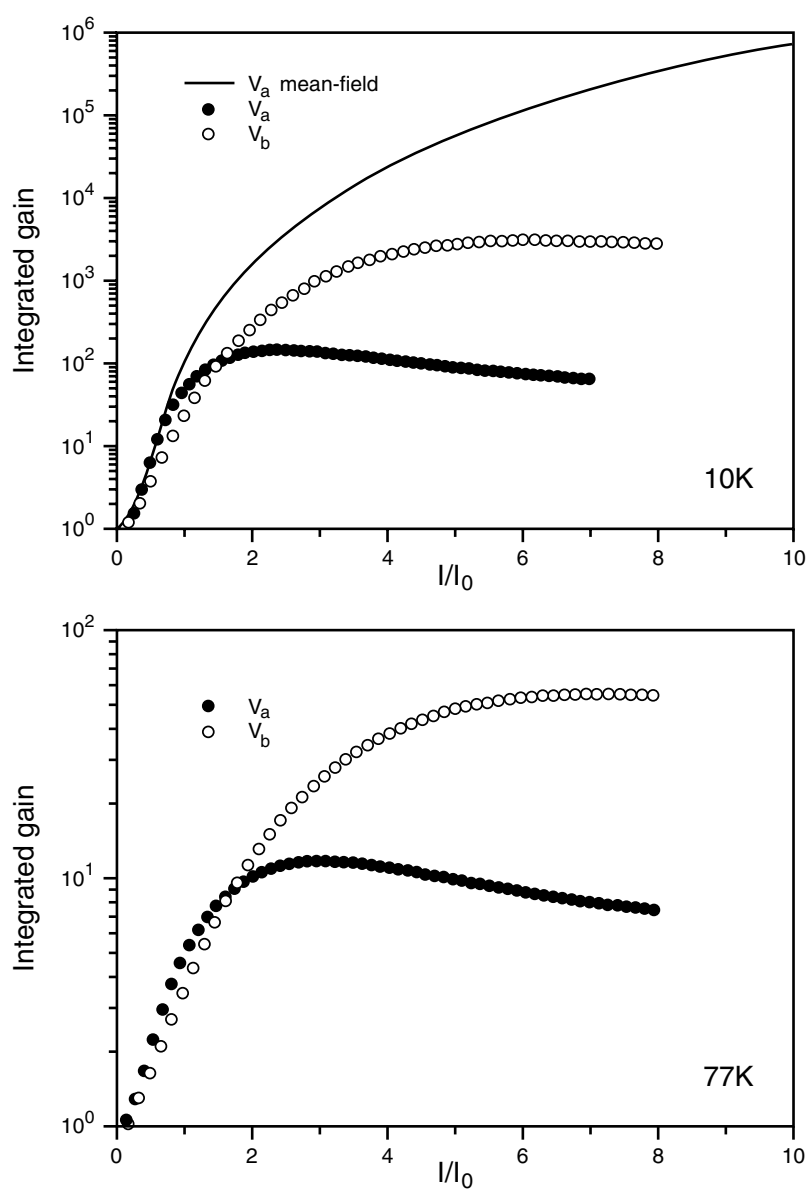

Figure 1 The power dependence of the integrated gain (the total light intensity transmitted in the signal direction divided by the intensity transmitted in the absence of the pump beam) calculated for two GaAlAs based samples with splitting $2 V_{i}=10.6 \mathrm{meV}$ (corresponding to $\left.N_{\text {eff }}=4\right)$ and $2 V_{2}=15 \mathrm{meV}\left(N_{\text {eff }}=8\right.$ ). $I_{0}=10^{13}$ photons per $\mathrm{cm}^{2}$ per pulse. The exciting light pulses have $250 \mathrm{fs}$ duration. The material parameters of the two samples are coincident except for $N_{\text {eff }}$. The decay rate of cavity photons through the mirrors $\gamma_{c}=0.25 \mathrm{meV}$ is that of GaAlAs structures considered in Ref. [3]. The $7 \mathrm{~nm}$ wide QWs have a binding energy $E_{b}=13.5 \mathrm{~nm}$. Calculations have been performed by using exciton homogeneous broadenings extracted from polariton linewidth measurements in GaAlAs structures [7], $2 \gamma_{x}=0.6 \mathrm{meV}$ at $T=10 \mathrm{~K}$ (a), and $2 \gamma_{x}=1.4 \mathrm{meV}$ at $\mathrm{T}=$ $77 \mathrm{~K}$ (b). The gain curves have been obtained fully optimizing incidence angles, central frequencies of the input light beams and cavity mode energy. Maximum gain occurs when the cavity mode energy is lower then the exciton energy by slightly more than half the polariton splitting

\section{Coherent trapping}

We notice that in principle the role of signal and idler polaritons can be reversed, so that we can stimulate the scattering process sending a weak resonant light pulse in the 
idler direction, thus observing amplification of idler polaritons as well as a strong emission at normal incidence. Owing to the axial symmetry of the polariton energy dispersion we may think to many different parametric processes satisfying energy and momentum conservation. In particular the cavity can be pumped by different beams all at the same magic angle $\theta_{p}$ and with the same energy but with different azimuthal angle $\phi$. In this case the in-plane pump momenta $\mathbf{k}_{j}$, have all the same modulus $k=\omega \sin \theta_{p} / c$ but different directions $\mathbf{k}_{j}=k\left(\cos \phi_{j}, \sin \phi_{j}\right)$. These different parametric processes give rise each to a corresponding distinct idler beam with momentum $2 \mathbf{k}$, but only one signal beam in the direction normal to the SMC.

Thus we may entangle many distinct parametric processes realizing a sort of parametric trap where many different processes are forced to produce scattering towards a common polariton state. So the emission at normal incidence is the result of many eventually interfering parametric processes.

We can seed the SMC with two pump laser pulses at $\mathbf{k}_{1}$ and $\mathbf{k}_{2}$ (1,2 pump modes), on the lower polariton dispersion. Then two laser pulses respectively at $2 \mathbf{k}_{1,2}(1,2$ idler modes) are sent in the SMC. If the pump laser pulses excite the polariton dispersion at the magic angle (hence $\left|\mathbf{k}_{1}\right|=\left|\mathbf{k}_{2}\right|=k$ ), the two idler beams stimulate two independent scattering process in which two (1 or 2 ) pump polaritons are scattered in the 1 or 2 idler mode and in a single (degenerate) signal mode at $\mathbf{k}=0$ with the prescribed energy conservation $2 \omega_{\mathbf{k}_{1,2}}=\omega_{2 \mathbf{k}_{1,2}}+\omega_{0}$. Each process above described is analogous to the parametric amplification process obtained in several experiments with the difference that in this situation the process is stimulated sending a weak light pulse in the idler directions [determined by $\left.\phi_{1,2}, \arcsin \left(2 k c / \omega_{2 k}\right)\right]$ instead of the signal one. We observe that this particular configuration determines two different contributions to the signal amplification due to the two distinct amplification processes. Our aim is to show that acting on the delay and phase of the ultrafast idler laser pulses it is possible to obtain the coherent control of the signal emission. From the Heisenberg equations of motion for the quantum polariton operators performing the rotating wave approximation and the taking the expectation values of the quantum operators involved, we obtain

$i \hbar \dot{p}_{0}=$

$=-i \Gamma_{0} p_{0}+g\left(p_{2 \mathbf{k}_{1}}^{*} p_{\mathbf{k}_{1}}^{2}+p_{2 \mathbf{k}_{2}}^{*} p_{\mathbf{k}_{2}}^{2}\right)+i C_{0} \sqrt{\gamma} E_{0}^{i n}$,

$i \hbar \dot{p}_{2 \mathbf{k}_{1}}=-i \Gamma_{2 k} p_{2 \mathbf{k}_{1}}+g \hat{p}_{0}^{*} p_{\mathbf{k}_{1}}^{2}+i C_{2 k} \sqrt{\gamma}$,

$i \hbar \dot{p}_{2 \mathbf{k}_{2}}=-i \Gamma_{2 k} p_{2 \mathbf{k}_{2}}+g \hat{p}_{0}^{*} p_{\mathbf{k}_{2}}^{2}+i C_{2 k} \sqrt{\gamma} E_{2 \mathbf{k}_{2}}^{i n}$,

where $g$ is the effective interaction parameter, $p_{j, \mathbf{k}}=$ $\left\langle p_{j, \mathbf{k}}\right\rangle, \Gamma_{i}$ and $C_{i}$ are respectively the dephasing, and the photon Hopfield coefficient relative to the i-th polariton branch; $E_{i}^{i n}$ describes the input light pulse exciting the i-th polariton branch; $\sqrt{\gamma}$ gives the beam fraction passing the cavity with equal mirror. In this approach the two pump beams are considered classical, having amplitudes $p_{\mathbf{k}_{1}}=\exp (-i \omega t) \tilde{p}_{\mathbf{k}_{1}}$ and $p_{\mathbf{k}_{2}}=\sqrt{\alpha} \exp (-i \omega t+i \phi) \tilde{p}_{\mathbf{k}_{1}}$, where $\alpha$ is a real constant. They are mutually coherent and proportional to the same classical amplitude, with a mutual difference of phase $\phi$ that can be achieved in experiment by a delay line. We assume a positive and real.

In order to obtain analytical simple expressions, we can assume for the input idler pulses an ultrafast deltalike behaviour:

$$
\left\{\begin{array}{l}
E_{2 \mathbf{k}_{1}}^{i n}=\epsilon \delta\left(t-t_{0}\right) \\
E_{2 \mathbf{k}_{2}}^{i n}=\epsilon e^{-i \omega} \delta\left(t-t_{0}-\delta\right)
\end{array}\right.
$$

and the case with $\alpha=1$ and in absence of delay between the two idler pulses $\delta=0$, we obtain:

$\tilde{p}_{0} \propto$

$\propto e^{-\Gamma\left(t-t_{0}\right)} \epsilon \Theta\left(t-t_{0}\right)\left\{1+e^{-i(\psi-2 \phi)}\right\} \sinh \left(\bar{\Delta}\left(t, t_{0}\right)\right.$,

$\tilde{p}_{2 k_{1}} \propto e^{-\Gamma\left(t-t_{0}\right)} \epsilon \Theta\left(t-t_{0}\right) \times$

$\times\left\{\cosh \left[\bar{\Delta}\left(t, t_{0}\right)\right]+1+e^{-i(\psi-2 \phi)}\left(\cosh \left[\bar{\Delta}\left(t, t_{0}\right)\right]-1\right)\right\}$,

$\tilde{p}_{2 k_{2}} \propto e^{-\Gamma\left(t-t_{0}\right)} \epsilon \Theta\left(t-t_{0}\right) \times$

$\times\left\{\cosh \left[\bar{\Delta}\left(t, t_{0}\right)\right]-1+e^{-i(\psi-2 \phi)}\left(\cosh \left[\bar{\Delta}\left(t, t_{0}\right)\right]+1\right)\right\}$.

We have defined

$\bar{\Delta}\left(t_{1}, t_{2}\right)=-i \tilde{g} \int_{t_{2}}^{t_{1}}\left(\left|\tilde{p}_{\mathbf{k}_{1}}\right|^{2}\left(t^{\prime}\right)\right) d t^{\prime} ; \quad \tilde{g}=i g \sqrt{1+\alpha^{2}}$.

We are also assuming that the pump polariton amplitudes $p_{\mathrm{k}}$ are not affected by the parametric process.

Eq. (11) show the possibility to obtain a complete coherent control at zero delay $(\delta=0)$ of emission at normal incidence acting on the phase differences $\psi-2 \phi$. Choosing e.g. the two pump beams with the same phase $\phi=0$, a complete stop of the amplification and emission can be obtained sending two idler beams at zero relative delay with a phase difference $\psi=\pi$. It is possible to show that almost complete coherent control can be achieved sending the two idler pulses with relative delay small as compared with the decay rate of polariton waves.

We can also observe that it is possible, seeding the SMC with two pump beams with opposite relative phase $\phi=n \pi$, to achieve destructive coherent control even if the phase of the two idler pulses is the same $(\psi=0)$. This configuration realizes the effect of parametric polariton trapping. This coherent trapping occurs due to destructive quantum interference between the two parametric processes contributing to the signal emission in the direction normal to the SMC. 


\section{Conclusions}

The results here shown clarify the origin of the great enhancement of parametric gain observed when increasing the polariton splitting. Observing that the wave-like nature of excitations in solids tells us that collisions between excitons are nothing but interference phenomena we are now able to affirm that the strong-coupling regime of SMCs, altering the phase of the interacting waves, provides a means to control the interference process. This coherent control of exciton-exciton interactions is able to produce highly desirable almost decoherence-free collisions that make possible to reach a very-high degree of amplification. Almost decoherence-free exciton-exciton interactions and hence structures with large polariton splitting are expected to be essential to reach room temperature operation.

Moreover the availability of decoherence-free collisions appears crucial for the quantum control and manipulation of the polariton wavefunction inside the cavity.

We have shown that it is possible, seeding the SMC with two pump beams with opposite relative phase, to achieve destructive coherent control even if the phase of the two idler pulses is the same. This configuration realizes the effect of parametric polariton trapping. This coherent trapping occurs due to destructive quantum interference between the two parametric processes contributing to the signal emission in the direction normal to the SMC

The parametric processes here analyzed and the possibility of controlling $X X$ collisions open new perspectives in the field of semiconductor quantum optics [15] and are promising for the realization of entangled collective polariton states for quantum information and computation.

\section{References}

[1] C. Weisbuch, M. Nishioka, A. Ishikawa, and Y. Arakawa, Phys. Rev. Lett. 69, 3314 (1992).

[2] P.G. Savvidis, J.J. Baumberg, R.M. Stevenson, et al., Phys. Rev. Lett. 84, 1547 (2000).

[3] M. Saba, C. Ciuti, J. Bloch, et al., Nature 414, 731 (2002).

[4] S. Savasta, O. Di Stefano, and R. Girlanda, Phys. Rev. Lett. 90, 096403 (2003).

[5] S. Savasta, O. Di Stefano, and R. Girlanda, Phys. Rev. A 64, 073306 (2001).

[6] E. Arimondo, Progress in Optics 35 (Elsevier, Amsterdam, 1996) p. 257.

[7] S. Savasta and R. Girlanda, Phys. Rev. Lett. 77, 4736 (1996).

[8] V. M. Axt and S. Mukamel, Rev. Mod. Phys. 70, 145 (1998).

[9] D.S. Chemla and J. Shah, Nature 411, 549 (2001).

[10] Th. Östreich, K. Schönhammer, and L.J. Sham, Phys. Rev. Lett. 74, 4698 (1995).

[11] S. Savasta and R. Girlanda, Phys. Rev. A 59, 15409 (1999).

[12] R. Takayama, N.H. Kwong, I. Rumyantsev, et al., Eur. Phys. J. B 25, 445 (2002).

[13] N.H. Kwong, R. Takayama, I. Rumyantsev, et al., Phys. Rev. Lett. 87, 027402 (2001).

[14] M.O. Scully and M.S. Zubairy, Quantum Optics (Cambridge University Press, Cambridge, 1997).

[15] S. Savasta, O. Di Stefano, V. Savona, and W. Langbein, in press. 